\title{
Computed Tomography Angiography of Gastrocolic Vein Trunk by Morphological Filtering Technique in Right Colon Cancer
}

This article was published in the following Dove Press journal: Therapeutics and Clinical Risk Management

\author{
Lei $\mathrm{Gu}^{\mathrm{l}}, *$ \\ Siyuan Wen ${ }^{1} * *$ \\ Chunjie $\mathrm{Xu}{ }^{\prime}$ \\ Jiong $\mathrm{Zhu}^{2}$ \\ Peilin Liu $^{3}$ \\ Qing $X u^{\prime}$
}

'Department of Gastrointestinal Surgery, Renji Hospital, School of Medicine Shanghai Jiao Tong University, Shanghai, People's Republic of China; ${ }^{2}$ Department of Radiology, Renji Hospital, School of Medicine Shanghai Jiao Tong University, Shanghai, People's Republic of China; ${ }^{3}$ School of Electronic Information and Electronic Engineering, Shanghai Jiao Tong University, Shanghai, People's Republic of China

*These authors contributed equally to this work
Correspondence: Qing Xu

Department of Gastrointestinal Surgery,

Renji Hospital, School of Medicine

Shanghai Jiao Tong University, Shanghai,

People's Republic of China

Email renjixuqing@163.com
Background: This study was to evaluate CT vein image segmentation and threedimensional imaging in the anatomical structure of gastrocolic venous trunk before operation for colon cancer.

Methods: CT scanning images by Philips Brilliance CT 256CT scanner. Vein position was segmented and calculated by grey value through algorithm flow. Intensity measurement of selected image area by a calculate through noise cancellation and missing pixel filling, based on numerical morphology.

Results: The direction of the right colonic vein could be clearly displayed in all 96 patients by morphological filtering technique. Among these patients, there were 78 patients with gastrocolic vein trunk, with an occurrence rate of $81.25 \%$. According to the classification of GVT, there were 36 cases of type A (46.2\%), 22 cases of type B (28.2\%), 12 cases of type $\mathrm{C}(15.4 \%)$ and 6 cases of type D (7.7\%).

Conclusion: CT vein image segmentation and three-dimensional imaging can effectively evaluate the anatomical variation of gastrocolic vein trunk before operation, which is helpful for operators to correctly understand its anatomical structure and choose a reasonable anatomical approach.

Keywords: colon cancer, computed tomography angiography, three-dimensional reconstruction, gastrocolic vein trunk, morphological filtering technique

\section{Introduction}

In the past two decades, complete mesocolic excision (CME) has been introduced into colon cancer surgery, which not only significantly improves the survival rate of patients with colon cancer, but also improves the operator's experience and technical level. ${ }^{1}$ Misperceptions of vascular abnormalities can lead to uncontrollable bleeding. In the resection of right colon cancer, the management of gastrocolic vein trunk (GVT) is more complex, and its branches are highly variable. ${ }^{2}$ A correct understanding of the anatomical structure of the gastrocolic vein trunk before operation is helpful for the operator to choose a reasonable approach for dissection, to reduce bleeding and improve the effect of operation.

The distribution of blood vessels in CT sequence images is an important basis for disease diagnosis, treatment plan and operation planning. The distribution of blood vessels can be extracted and displayed automatically by image segmentation technology and 3D modeling technology, which can provide doctors with more intuitive and accurate diagnostic information. ${ }^{3}$ The purpose of this study was to 
evaluate the anatomical structure of gastrocolic venous trunk before operation for colon cancer by morphological filtering technique for three-dimensional reconstruction, and to explore its clinical value.

\section{Materials and Methods General Information}

In this study, 96 patients with right colon cancer were treated by laparoscopy in Renji Hospital, Shanghai Jiao Tong University School of Medicine in 2019, including 58 males and 38 females, aged from 27 to 82 years old, with a median age of 61 years. The study was approved by the Research Ethics Committee of Renji Hospital and carried out in accordance with the ethical standards formulated in the Helsinki Declaration. All patients provided their informed consent.

\section{Method}

\section{CT Scanning Method}

Plain and enhanced abdominal scans were performed with Philips Brilliance CT 256CT scanner. The layer thickness is $5 \mathrm{~mm}$. Pitch 0.800 , rack speed $0.5 \mathrm{~s} /$ week, matrix $512 \times 512$, tube voltage $120 \mathrm{kV}$, tube current $150 \mathrm{mAs}$. The contrast agent Iopromide $0.37 \mathrm{~g} / \mathrm{L}$ was injected into the enhanced scan with an injection flow rate of $5.0 \mathrm{~mL} / \mathrm{s}$, and a dose of $90 \mu \mathrm{g} / 100 \mathrm{~mL}$. Delayed 29s and 35s arterial phase and venous phase scanning.

\section{Vein Segmentation and 3D Reconstruction}

In CT image, the gray value of vein position is obviously different, which can be segmented by gray intensity. But in the actual image sequence, in order to track the vein region reliably, several factors need to be considered, including: 1) the "noise" near the segmentation area caused by interfering pixels in the CT image; 2) the gray change of the vein region in the adjacent frame; 3) the offset of the vein center position in the adjacent frame image; and 4) the vein shape change. In order to solve the above problems, we use a frame-by-frame adaptive dynamic threshold image segmentation algorithm and a contour tracking algorithm that supports continuous deformation to ensure continuous tracking of the changing vein direction in the CT image. The specific process of this method is shown in Figure 1

The specific explanation of the process shown in the above figure is as follows: in the 3D tracking initialization phase, the vein tracking process in the image frame is started by manually selecting the center of the area to be

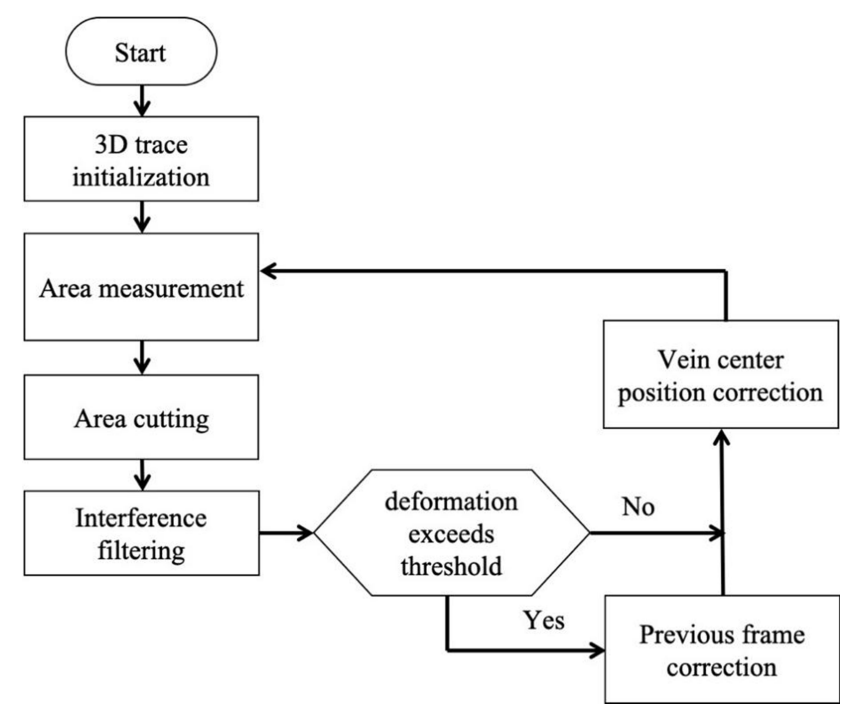

Figure I Algorithm flow.

tracked. In the following process, the algorithm is completed in turn:

1. Intensity measurement of selected image area

Calculate $\theta=\frac{1}{\mathrm{~N}} \sum_{\mathrm{p}_{\mathrm{n}} \in \mathrm{D}} \mathrm{I}\left(\mathrm{P}_{\mathrm{n}}\right)$, where $\mathrm{D}$ represents the selected image area, $p_{n}$ is the pixel coordinates in the area, $N$ is the area area, and $I\left(p_{n}\right)$ is the brightness of the image frame corresponding to the pixel at the $\mathrm{p}_{\mathrm{n}}$ position.

2. Region cutting based on intensity threshold, which divides the pixels whose brightness is greater than that in the image $\theta$.

3. Interference filtering based on numerical morphology.

Due to noise and image interference, the actual segmentation effect is not ideal, as shown in Figure 2

In order to improve the quality of contour shape extraction, we use morphological filtering algorithm. The main feature of this algorithm is that it can keep the effective area of the filtered object unchanged. The noise points are deleted by morphological open operation and the missing pixel filling is compensated by morphological closed operation. The effect of these two steps is shown in Figure 3

In addition, because there are other pixels in the image that exceed the threshold, they can be regarded as interference patterns. We delete them through the "area" growth algorithm, that is, use the $c_{t-1}$ coordinates of the vein area center of the previous frame as the "seed", and 


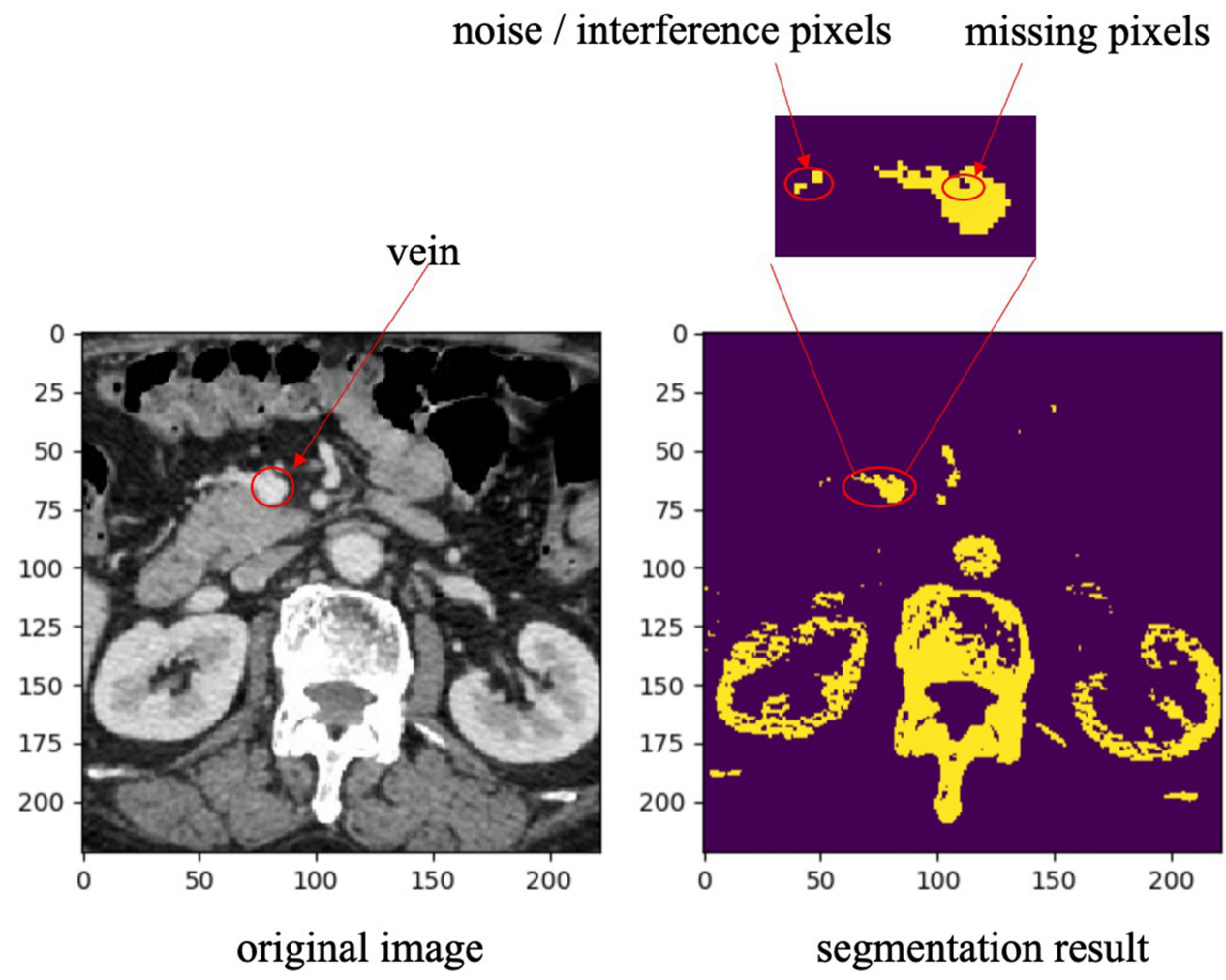

Figure 2 Simple threshold segmentation result.
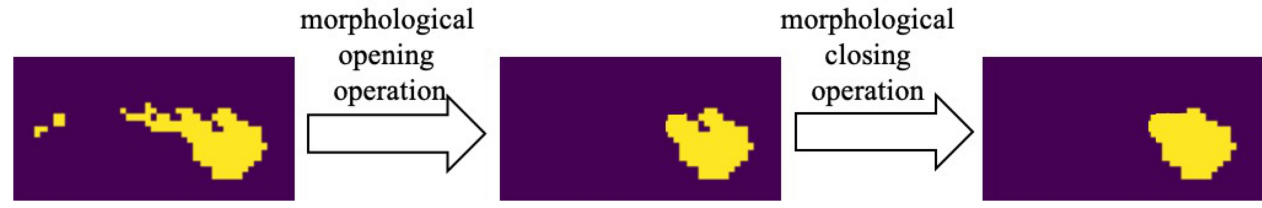

Figure 3 Noise cancellation and missing pixel filling based on numerical morphology.

surround the "seed" selects its connected domains through regional growth and deletes all non-connected parts.

4. Deformation estimation based on geometric parameters of contours.

The quantitative calculation of contour geometric deformation is based on the ellipse fitting of the current segmented image region, and the ratio of the long and short sides of the ellipse is used as the deformation parameter. We use the pixel coordinates $p_{n}$ (expressed as $2 \times 1$ column vector) in region $\mathrm{D}$ to construct the covariance matrix of $4 \times 4: C=\sum_{n}\left(P_{n}-\bar{P}\right)\left(P_{n}-\bar{P}\right)^{T}$, where $\stackrel{-}{P}$ is the average coordinates of pixels in the region. The ratio $\eta=\sigma_{\max } / \sigma_{\min }$ between the maximum $\sigma_{\max }$ and the minimum eigenvalue $\sigma_{\min }$ of $\mathrm{C}$ is used as the geometric deformation parameter. For a circle, $\eta$ is close to 1 , for ellipse, $\eta$ is greater than 1 , and the more "flat length", the larger $\eta$.
By comparing the difference of parameter $\eta$ between two adjacent frames, we can judge whether there is shape mutation. Because we assume that the distance difference between two adjacent CT images is very small, the vein shape does not allow mutation. Therefore, when there is a sudden change, it is considered that the current image segmentation is not available, and therefore enter the contour correction operation.

1. Contour correction-this step is used to correct the shape mutation error that occurred during tracking by replacing the current region profile with the region outline of the previous frame.

2. Correction of the central position of the vein

In this paper, two cases of central venous correction are considered. One is that the vein contour changes suddenly, and the current contour copies the results of the previous frame. 
Because of the specific movement of veins between different CT frames, we not only copy the images, but also consider the continuous movement characteristics of the vein center. That is, the shape of the current vein contour is the same as that of the previous frame, but the central position is different. Specifically, the central position $c_{t}$ is calculated by the following formula: $c_{t}=c_{t-1}+\left(c_{t-1}-c_{t-2}\right)$, in which $c_{t-1}$ and $c_{t-2}$ are the central positions of vein contour in the first two frames respectively. The other case is that the current contour is acceptable, and the pixel coordinates mean value of the current segmentation area is used as the new vein center. As shown in Figure 4:

Through the above algorithm flow, we can track the vein direction from the continuous image frames and extract the complete 3D vein space point cloud coordinates.

\section{Description of Variation of Gastrocolic Venous Trunk}

The gastrocolic vein trunk, also known as the Henle trunk, has an anatomical occurrence rate of $45.9-100 \%{ }^{4-6}$ Its branches include right gastroepiploic vein (RGEV), anterior superior pancreaticoduodenal vein (ASPDV), right colonic vein, (RCV), superior right colonic vein (SRCV) and middle colonic vein (MCV). According to the branch variation classification of $\mathrm{Jin}^{7}{ }^{7}$ it can be divided into four types: type A: RGEV+ASPDV+SRCV; type B: RGEV +ASPDV+SRCV+RCV; type C: RGEV+ASPDV+SRCV $+\mathrm{MCV}$ and type D: RGEV+ASPDV. RGEV and ASPDV are relatively fixed branches. (Figure 5)

\section{Result}

\section{The Validity of Angiography of GVT by Morphological Filtering Technique}

All cases can be manually identified by CT images, and 3D images can be exported by software. Through vein

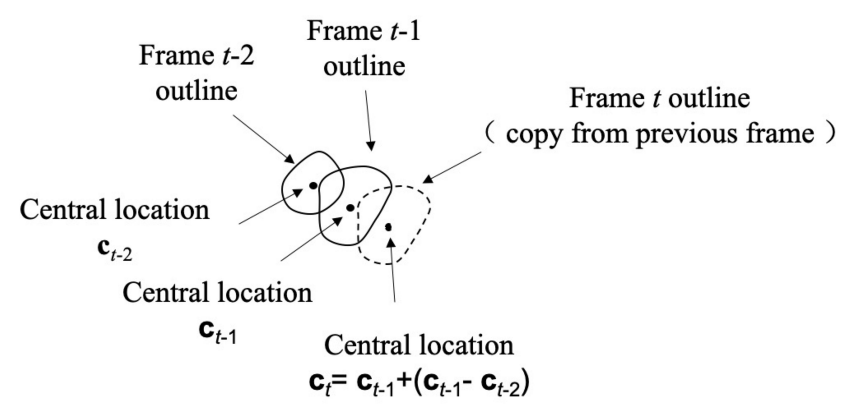

Figure 4 When the vein outline is copied from the previous frame, the outline center is calculated. image segmentation and three-dimensional reconstruction by morphological filtering technique, the direction of the right colonic vein could be clearly displayed in all 96 patients. (Figure 6)

\section{Occurrence Rate of Gastrocolic Venous Trunk}

Among the 96 patients, there were 78 patients with gastrocolic vein trunk, with an occurrence rate of $81.25 \%$.

\section{Variation of Branches of Gastrocolic Venous Trunk}

According to the classification of GVT, there were 36 cases of type A (46.2\%), 22 cases of type B (28.2\%), 12 cases of type C (15.4\%) and 6 cases of type D (7.7\%). There were 2 patients whose RGEV, ASPDV and MCV were imported into GVT, and were not included in this classification system.

\section{Discussion}

Gastrocolic venous trunk (GVT) is the confluence point of RGEV and ASPDV, which was first proposed by Henle ${ }^{8}$ in 1868, also known as Henle trunk. The average caliber (SMV) of GVT converging into the superior mesenteric vein along the inferior margin of the pancreas was $5.2 \mathrm{~mm} .{ }^{9}$ GVT is an important anatomical marker between hepatic flexure and transverse colon. ${ }^{10}$

The clinical significance of preoperative evaluation of GVT anatomical structure before resection of right colon cancer is mainly reflected in two aspects: one is that it becomes the main cause of intraoperative bleeding because of the diversity and complexity of its branches. During laparoscopic surgery, due to the lack of tactile perception, the intraoperative bleeding rate is relatively high if vascular anatomy is not evaluated in advance, it may cause uncontrollable bleeding. ${ }^{11}$ Second, because of the particularity of its location, it has become an important anatomical sign in the process of lymphatic dissection of right colon cancer. JSCCR put forward the concept of D3 lymph node dissection in the $1980 \mathrm{~s},{ }^{12}$ and correct D3 lymph node dissection requires the operator to correctly identify and separate SRVC in the resected area in order to reduce intraoperative bleeding.

The branches of gastrocolic vein trunk mainly include RGEV, ASPDV and colonic vein, in which the anatomical variation is mainly on the colonic vein. Among them, the main type of genus-branch variation is RGEV+ASPDV 


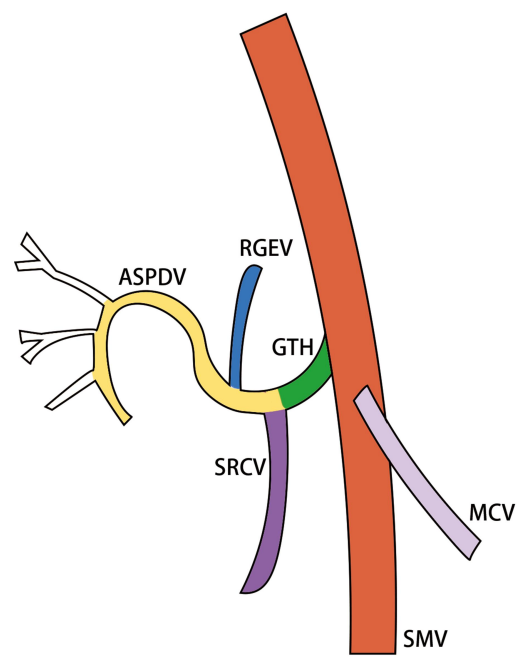

A

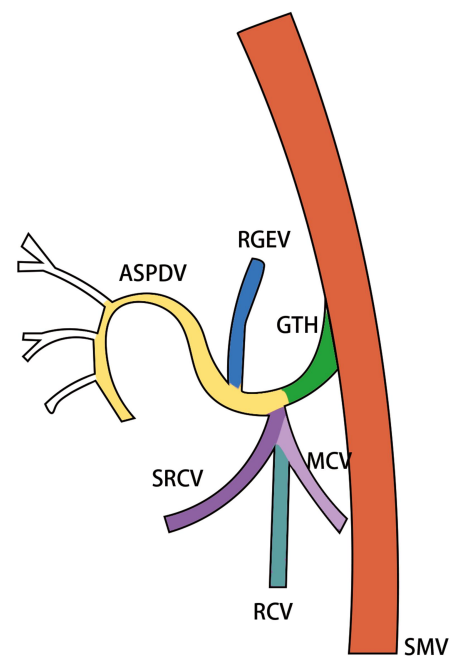

C

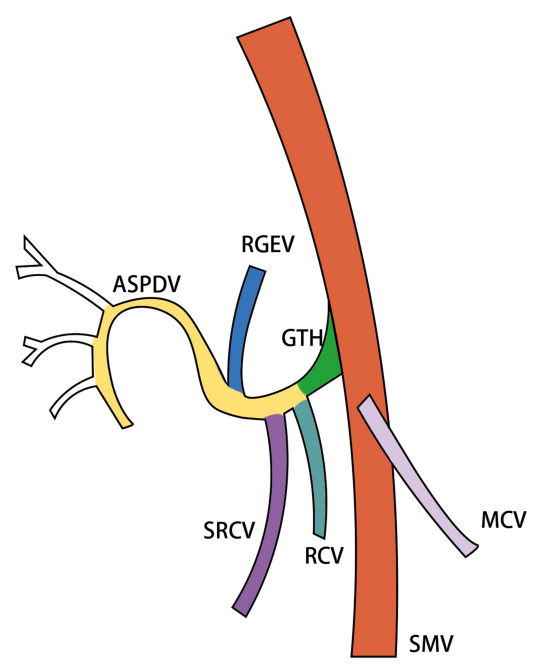

B

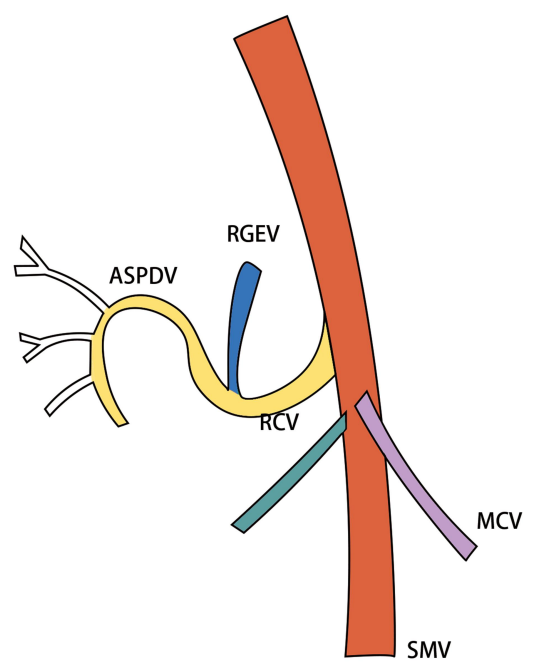

D

Figure 5 Branch variation classification of GVT: Type (A) RGEV+ASPDV+SRCV; Type (B) RGEV+ASPDV+SRCV+RCV; Type (C) RGEV+ASPDV+SRCV+MCV; Type (D) RGEV+ASPDV.

$+\mathrm{SRCV}$, with a frequency of about $34.6 \% .{ }^{13}$ In this study, the occurrence rate of this variant type is $46.2 \%$, which is basically consistent with related research. At present, there is no unified classification method for the variation of the branches of the gastrocolic vein trunk, and some Japanese scholars have proposed to classify the anatomical variation of the gastrocolic vein trunk into $0 \sim$ III types according to the number of branches of the gastric colonic vein.

The evaluation methods of gastrocolic venous trunk mainly include autopsy, intraoperative anatomy and imaging evaluation. ${ }^{14}$ Computed tomography angiography, magnetic resonance imaging (MRI) and Doppler ultrasound are available imaging approaches to evaluate vascular anatomy. Compared with other imaging evaluation methods, 3D CT is lower cost, inconvenience and risk to patients. ${ }^{15}$ Previous experience proves 3D-CT angiography is a non-invasive, convenient and accurate method to evaluate variation of vessel in gastrointestinal surgery. ${ }^{16,17}$ The imaging preacademic method CT vein image segmentation and threedimensional reconstruction that used in this study is affected by intravenous contrast agent, scanning method and scanning collimation, so it still has some limitations. Limited by the length of ASPDV, it is difficult to find the branches of ASPDV accurately in some imaging studies. ${ }^{18}$ 


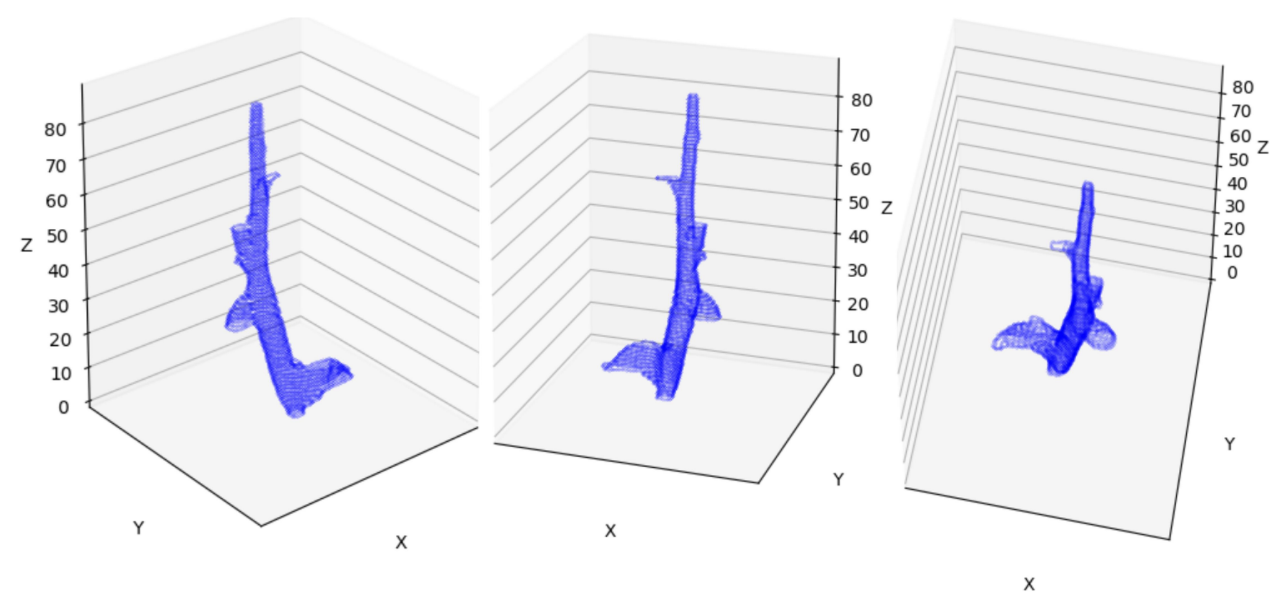

Figure 6 3D reconstruction of SMV system.

In the operation of right colon cancer, according to the CME principle, lymphatic dissection should be carried out along the gastrocolic vein trunk. Because of the high variability of the gastrocolic vein trunk, the operator should choose a reasonable approach to avoid bleeding. In the early stage, the lateral approach it is easy to pull the hepatic flexure of the colon and cause GVT bleeding. ${ }^{19}$ At present, the intermediate approach is the main anatomical method of laparoscopic radical resection of colon cancer, ${ }^{20}$ but when dissecting upward along SMV, it is still easy to cause injury of GVT and pancreas. In recent years, anatomical methods such as cephalic approach, combined approach and paging approach have been introduced, and the operator can choose a reasonable approach according to the preoperative evaluation of gastrocolic venous trunk.

In summary, CT vein image segmentation and threedimensional imaging can effectively evaluate the anatomical variation of gastrocolic vein trunk before operation, which is helpful for operators to correctly understand its anatomical structure and choose a reasonable anatomical approach. In addition, the study of comparison between intraoperative anatomy and preoperative CTA of GVT is in progress.

\section{Funding}

This work was supported by Research Fund for medical engineering cross project of Shanghai Jiao Tong University (ZH2018ZDA08).

\section{Disclosure}

The authors report no conflicts of interest for this work.

\section{References}

1. West NP, HohenbergerW Weber K, Weber K, Perrakis A, Finan PJ, Quirke P. Complete meso colic excision with central vascular ligation produces an oncologically superior specimen compared with standard surgery for carcinoma of the colon. J Clin Oncol. 2010;28:272-278. doi:10.1200/JCO.2009.24.1448

2. Alsabilah JF, Razvi SA, Albandar MH, Kim NK. Intraoperative archive of right colonic vascular variability aids central vascular ligation and redefines gastrocolic trunk of henle variants. Dis Colon Rectum. 2017;60:22-29. doi:10.1097/DCR.0000000000000720

3. Miyazawa M, Kawai M, Hirono S, et al. Preoperative evaluation of the confluent drainage veins to the gastrocolic trunk of Henle: understanding the surgical vascular anatomy during pancreaticoduodenectomy. J Hepatobiliary Pancreat Sci. 2015;22:386-391. doi:10.1002/jhbp.205

4. Matsuki M, Tanikake M, Kani H, et al. Dual-phase 3D CT angiography during a single breath-hold using 16-MDCT: assessment of vascular anatomy before laparoscopic gastrectomy. Am J Roentgenol. 2006;186:1079-1085. doi:10.2214/AJR.04.0733

5. Spasojevic M, Stimec B. Can the gastrocolic trunk of henle serve as an anatomical landmark in laparoscopic right colectomy?A postmortem anatomical study. Am J Surg. 2010;199:249-254. doi:10.1016/j. amjsurg.2009.03.010

6. Lange JF, Koppert S, van Eyck CH, Kazemier G, Kleinrensink GJ, Godschalk M. The gastrocolic trunk of Henle in pancreatic surgery: an anatomo-clinical study. $J$ Hepatobiliary Pancreat Surg. 2007;7:401-403. doi:10.1007/s005340070035

7. Jin G, Tuo H, Sugiyama M, et al. Anatomic study of the superior right colic vein: its relevance to pancreatic and colonic surgery. $\mathrm{Am}$ J Surg. 2006;191(1):100-103. doi:10.1016/j.amjsurg.2005.10.009

8. Henle J. Handbook, 1968. The systematic anatomic of humans.III.1: Handbuch der gefaesslehre des Menschen Note 1 rMl.Braunschweig. Germany: Friedrich vieweg und sohn; 1968:371.

9. Ignjatovic D, Stimec B, Finjord T, Bergamaschi R. Venous anatomy of the right colon: three-dimensional topographic mapping of the gastrocolic trunk of Henle. Tech Coloproctol 8. 2004;19-21:discussion 21-22. doi:10.1007/s10151-004-0045-9

10. Mori S, Baba K, Yanagi M, et al. Laparoscopic complete mesocolic excision with radical lymph node dissection along the surgical trunk for right colon cancer. Surg Endosc. 2015;29:34-40. doi:10.1007/ s00464-014-3650-3

11. Peltrini R, Luglio G, Pagano G, Sacco M, Sollazzo V. Luigi Bucci Gastrocolic trunk of Henle and its variants: review of the literature and clinical relevance in colectomy for right-sided colon cancer. Surg Radiol Anat. 2019;41:879-887. doi:10.1007/s00276-019-02253-4 
12. Okuno K. Surgical treatment for digestive cancer. Curr Issues Colon Cancer Dig Surg. 2007;24:108-114. doi:10.1159/000101897

13. Gao Y, Lu Y. Variations of gastrocolic trunk of henle and its significance in gastrocolic surgery. Gastroenterol Res Pract. 2018;6:3573680. doi:10.1155/2018/3573680

14. Alsabilah J, Kim WR, Kim NK. Vascular structures of the right colon: incidence and variations with their clinical implications. Scand J Surg. 2017;106(2):107-115. doi:10.1177/1457496916650999

15. Bogetti JD, Herts BR. Accuracy and utility of 3-dimensional computed tomography in evaluating donors for adult living related liver transplants. Comparative Study Liver Transpl. 2001;(8):687-692. doi: $10.1053 / \mathrm{jlts.} 2001.26351$

16. Ogino T, Takemasa I, Horitsugi G, et al. Preoperative evaluation of venous anatomy in laparoscopic complete mesocolic excision for right colon cancer. Ann Surg Oncol. 2014;21(S3):429-435. doi:10.1245/s10434-014-3572-2

17. Cai J, Wen X, Lin W, et al. Evaluation of three-dimensional CT reconstruction on the anatomic variation of inferior mesenteric artery and left colic artery. Zhonghua Wei Chang Wai Ke Za Zhi. 2017;20:1274-1278.
18. Mehmet Ayhan Kuzu, Erkin İsmail, Safa Çelik, Muhammed Feyzi Şahin, Mehmet Ali Güner, Werner Hohenberger, Halil İbrahim Açar. Variations in the vascular anatomy of the right colon and implications for right-sided colon surgery. Dis Colon Rectum. 2017;3:290-298. doi:10.1097/DCR.0000000000000777

19. Sakaguchi T, Suzuki S, Morita Y, et al. Analysis of anatomic variants of mesenteric veins by 3-dimensional portography using multidetector-row computed tomography. Am J Surg. 2010;200:1522. doi:10.1016/j.amjsurg.2009.05.017

20. Feng B, Yan X, Zhang S, Xue P, Zirui H, Zheng M. Anatomical strategies of Henle trunk in laparoscopic right hemi-colectomy for right colon cancer. Zhonghua Wei Chang Wai Ke Za Zhi. 2017;20:635-638.

\section{Publish your work in this journal}

Therapeutics and Clinical Risk Management is an international, peerreviewed journal of clinical therapeutics and risk management, focusing on concise rapid reporting of clinical studies in all therapeutic areas, outcomes, safety, and programs for the effective, safe, and sustained use of medicines. This journal is indexed on PubMed Central, CAS,
EMBase, Scopus and the Elsevier Bibliographic databases. The manuscript management system is completely online and includes a very quick and fair peer-review system, which is all easy to use. Visit http://www.dovepress.com/testimonials.php to read real quotes from published authors. 